Médiation du discours expert : approches linguistiques

\title{
Cécile Desoutter et Caroline Mellet (dir.), Le discours rapporté : approches linguistiques et perspectives didactiques
}

Berne : Peter Lang, Linguistic Insights 178, 2013

\section{Geneviève Bordet}

\section{(2) OpenEdition}

Journals

Édition électronique

URL : http://journals.openedition.org/asp/4166

DOI : 10.4000/asp.4166

ISSN : 2108-6354

\section{Éditeur}

Groupe d'étude et de recherche en anglais de spécialité

\section{Édition imprimée}

Date de publication : 1 mars 2014

Pagination : 125-129

ISSN : 1246-8185

Référence électronique

Geneviève Bordet, «Cécile Desoutter et Caroline Mellet (dir.), Le discours rapporté : approches

linguistiques et perspectives didactiques », ASp [En ligne], 65 | 2014, mis en ligne le 15 mars 2014 consulté le 05 novembre 2020. URL : http://journals.openedition.org/asp/4166 ; DOI : https://doi.org/ $10.4000 /$ asp.4166

Ce document a été généré automatiquement le 5 novembre 2020.

Tous droits réservés 
Cécile Desoutter et Caroline Mellet (dir.), Le discours rapporté : approches linguistiques et perspectives didactiques

Berne : Peter Lang, Linguistic Insights 178, 2013

Geneviève Bordet

\section{RÉFÉRENCE}

Desoutter, Cécile et Caroline Mellet (dir.). 2013. Le discours rapporté : approches

linguistiques et perspectives didactiques. Berne : Peter Lang, Linguistic Insights 178. 270

pages. ISBN : 978-3-0343-1292-9. 
1 Cet ouvrage collectif, dirigé par Cécile Desoutter, de l'Université de Bergame (Italie) et Caroline Mellet, de l'Université Paris Ouest Nanterre, s'appuie sur des communications présentées à un colloque organisé à Bergame en 2011 sur le thème «Le discours rapporté et ses marques: perspectives théoriques et didactiques ". Les contributions portaient sur les marques du "discours autre ", direct ou indirect. L'objectif du colloque, et de l'ouvrage qui comprend un avant-propos, une introduction et douze chapitres, est double. Il s'agit d'abord d'analyser les difficultés que pose l'identification et le marquage des formes du discours rapporté, dans divers types de discours, et d'en comprendre les implications pour l'interprétation $\mathrm{du}$ texte. On attend

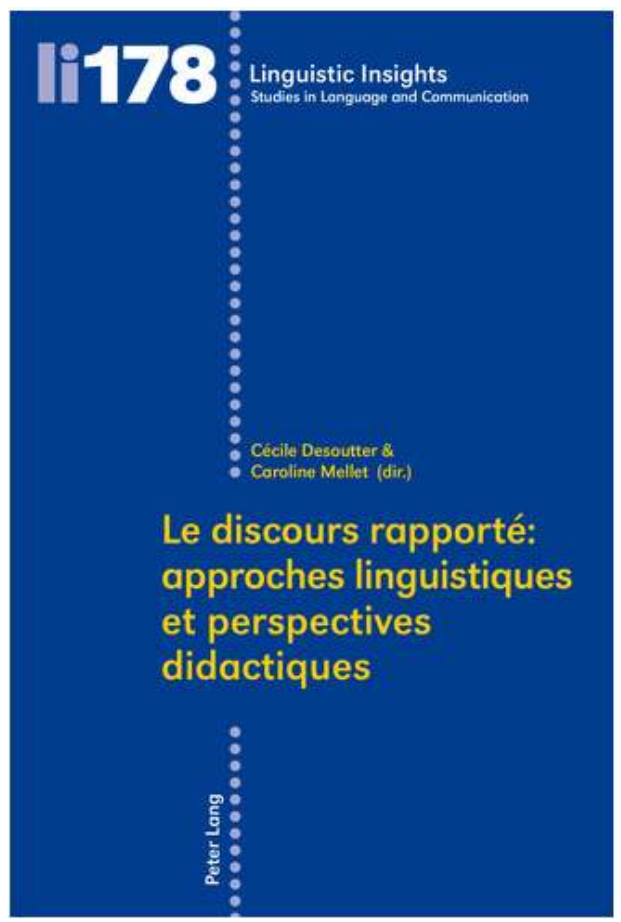
également de cette analyse qu'elle contribue à développer un outil didactique, le logiciel Navilire ${ }^{1}$, qui s'appuie sur l'analyse de corpus pour ouvrir aux lecteurs des pistes de navigation dans les textes. Ce projet est coordonné par Lita Lundquist de l'Université de Copenhague, également auteur de l'introduction de l'ouvrage. Les corpus annotés permettent de proposer aux étudiants des exercices où ils repèrent les structures linguistiques qui régissent la logique du texte.

Chaque contribution apporte à la fois des éléments pour une meilleure compréhension des formes et $\mathrm{du}$ rôle du discours rapporté dans le texte, et pour une application didactique dans le cadre du logiciel. Cette ligne directrice très claire permet l'ouverture à toutes formes de discours et de textes : oral, écrit, audiovisuel ; textes journalistiques, fiction, articles de recherche, documents procéduraux (comptes-rendus de réunions universitaires)... Les perspectives adoptées sont également extrêmement variées: expérimentale et didactique avec l'étude de la transcription d'un récit par des enfants ; tournée vers le discours scientifique et l'insertion des sources par les chercheurs; approche plus théorique sur les introducteurs de discours rapporté ; contrastive entre France et Italie dans un cas, entre articles rédigés en anglais par des locuteurs natifs et non natifs dans l'autre. On notera également que deux chapitres sont rédigés en italien, des résumés bilingues italiens-français étant présentés pour toutes les contributions.

3 Les références théoriques partagées renvoient aux thèmes du dialogisme et de la polyphonie (Bakhtine 1970; Ducrot 1984) et surtout de la théorisation de la « représentation du discours autre » (RDA) par Jacqueline Authier-Revuz. Les grands axes de réflexion portent sur la difficulté d'identification claire des marques du discours rapporté et la possibilité d'établir un continuum de discours rapporté du « marqué » au " non marqué ». La délimitation de l'ouverture et surtout de la clôture $\mathrm{du}$ discours rapporté apparaît problématique, ce qui fait obstacle à l'annotation de corpus à visée didactique. Cette difficulté amène plusieurs auteurs à proposer des typologies de segments introductifs, et notamment les verbes, qui permettent 
l'élaboration d'exercices automatisés de repérage du discours rapporté. Plus globalement, tous les auteurs s'intéressent à la palette de moyens d'expression du discours "autre» ou rapporté qu'ouvre le recours souvent combiné aux diverses formes du discours direct, indirect et indirect libre. Est surtout abordée la manière dont ce dispositif linguistique permet à la fois la mise en scène et l'identification des diverses strates énonciatives qui constituent le texte et guident la logique de lecture.

Chaque auteur propose une combinaison spécifique d'approche théorique, de choix de corpus, et de pistes d'application. Ainsi Denis Le Pesant analyse les verbes introducteurs de discours rapporté au style direct à partir de deux hypothèses. D'une part, ces verbes sont soit intransitifs soit utilisés avec une valeur intransitive. D'autre part, la relation de prédication entre le syntagme nominal sujet et le discours rapporté n'est pas assurée par des moyens lexicaux mais par des marquages typographiques, prosodiques, par le choix du temps des verbes et la position du sujet dans la phrase. Le choix lexical du verbe va en revanche donner au discours rapporté sa valeur émotionnelle et illocutoire. Il propose une typologie des verbes à connotation émotionnelle (communication, psychologie, affect...), élaborée à partir du dictionnaire Dubois-Charlier (1993). Cette typologie peut être utilisée pour une annotation de corpus visant à l'identification de la valeur sémantique des segments de discours rapporté.

5 Cécile Desoutter s'intéresse également au rôle des verbes dans son étude consacrée aux procès-verbaux de réunion de conseils de «facultés » en Italie et en France. Elle y analyse les systèmes d'insertion du discours rapporté en fonction des séquences concernées dans le procès-verbal telles qu'introduction, débats, clôture. Sont étudiés le rôle des verbes de parole ainsi que les marques typographiques qui, parce qu'elles portent sur l'enchâssement des différents types de discours rapporté, permettent de positionner le locuteur responsable du procès-verbal par rapport au locuteur dont les propos sont rapportés. L'observation de la manière dont sont ainsi théâtralisés les débats permet une approche comparative entre ce type de discours en Italie et en France, particulièrement quant à la place qu'il fait respectivement au rapporteur et au locuteur « rapporté ».

6 Caroline Mellet et Frédérique Sittri étudient des comptes-rendus de conseils universitaires en France. L'identification de la représentation du « discours autre » se heurte au problème des formes dites "non marquées " par exemple dans les phrases faisant suite à une séquence de discours indirect. L'assignation de l'origine de la parole repose sur un faisceau d'indices : indices compositionnels, sémantiques, de genre et de cohésion. La recherche met en évidence l'importance de la connaissance des règles liées à ce type de situation de communication, donc du genre, pour une interprétation pertinente des différents niveaux d'énonciation. Des exercices de repérage des différents types d'indices sont proposés.

7 D'autres contributions traitent du cas où il s'agit d'étayer une argumentation sur la citation de la parole d'un autre locuteur. C'est le cas de celle de Shirley Carter-Thomas et Elisabeth Rowley-Jolivet qui envisagent le cas du chercheur francophone confronté à la citation d'autres auteurs dans un article de recherche rédigé en anglais. À partir d'un triple corpus d'articles de recherche (écrits en anglais et en français par des locuteurs natifs, en anglais par des francophones), les auteurs observent la manière dont, à travers la pratique de la citation, le chercheur intègre dans son propre discours celui de l'« autre», en l'occurrence celui de chercheurs reconnus du domaine. Une approche contrastive permet d'identifier ce qui pose problème dans cette fonction 
essentielle de l'article de recherche pour des locuteurs non natifs. Elle met en évidence trois zones de difficulté: le choix des verbes introducteurs de "that» relatif; le fonctionnement différent de "according to" et "selon» ainsi que le calque non pertinent en anglais de l'usage du conditionnel en français pour marquer la distance avec l'auteur cité. L'analyse permet de proposer des exercices de repérage et de reformulation de la parole de l'autre.

De manière très complémentaire, Fanny Rinck propose un scénario didactique pour les apprentis-chercheurs confrontés à la gestion de la citation des sources. La technique de citation dans l'introduction de l'article de recherche est analysée en tant qu'intégration de la parole de l'autre et référence au savoir déjà existant ou "déjà là » au sens bakhtinien du terme. L'auteur établit une typologie des modalités de citation et la met en œuvre dans un scénario didactique fondé sur l'observation de corpus. L'objectif de l'exercice est de mettre en évidence le lien entre les modalités de citation et le type de stratégie rhétorique adoptée dans l'introduction.

9 Toujours dans la perspective de la citation mais avec un corpus très divers, Julie Lefebvre considère la note de bas de page comme indice et marqueur d'une RDA. La note de bas de page est prise comme "structure bilinéaire " et "chaîne graphique " articulée au corps du texte. À partir d'un corpus extrait de textes «occidentaux contemporains » des cinquante dernières années dans des genres discursifs divers, elle s'attache à décrire une progression des formes de marquage « fortes » vers des formes "faibles ", marques qui font de la note la trace de la présence d'une parole autre dans le texte.

10 C'est à la recherche de ce continuum également que s'attache Emilia Calaresu qui identifie les marques "indiscrètes" d'une "représentation et reconstruction de discours » (RRD), en s'appuyant sur une typologie des divers types de polyphonie, avec et sans discours rapporté (Carel \& Ducrot 2009). Elle décrit l'utilisation d'« extensions parasitaires » telle que «like » en anglais, " genre » ou «style » en français, ou « tipo » en italien, pour introduire la représentation d'un discours rapporté ; elle met aussi en évidence la difficulté à identifier automatiquement les marques de l'existence d'une RRD, dans un continuum qui va des marques « explicites » aux marques « implicites».

11 La place du «discours rapporté » dans la fiction sous toutes ses formes donne lieu à plusieurs types de réflexion. Ainsi, l'œuvre de Naguib Mahfouz, et plus particulièrement sa Trilogie du Caire, donne l'occasion à Leda Mansour de se pencher sur la question de l'utilisation du "discours rapporté » pour exprimer la parole "empêchée » ou "non prononcée " de personnages dont l'expression est parfois censurée, voire interdite. Elle établit une typologie des discours non prononcés, distinguant ainsi objet de souvenir, de sentiment, ou discours direct non prononcé, à partir de l'analyse des segments introducteurs. Elle propose de préférer le terme de " parole intérieure » à celui de " pensée intérieure », dans la mesure où « le narrateur [...] extériorise la vie intérieure de ses personnages » (p. 60).

12 C'est aussi à partir d'un corpus de fiction, des nouvelles de Giorgio Pagani, que Paola Polito et Erling Strudsholm analysent la manière dont est traduite textuellement grâce au discours rapporté l'expression non verbale et gestuelle des personnages. Une typologie des marques transcrivant une " sémiosis corporelle » est ensuite testée pour l'étude de textes d'Alessandro Manzoni, Carlo Collodi et Giovanni Verga.

13 Passant du texte au film, Francesca Pagani fait le pari audacieux de proposer un scénario didactique dans le cadre de Navilire à partir des versions comparées du 
scénario écrit et de la réalisation d'un film de Godard. Le scénario original constitue un " palimpseste » de discours rapportés, Godard utilisant un documentaire de Truffaut pour en proposer une version narrativisée grâce à un entrelacs de citations directes et de discours rapporté. L'étude des systèmes de marquage des niveaux d'énonciation permet d'explorer la manière dont se construit pour chaque version du récit une cohérence spécifique dans un texte fondé sur l'art de la digression. Les résultats de cette réflexion ouvrent la voie à la proposition de " parcours pédagogiques » mettant en lumière les pistes de cohérence à l'intérieur d'une logique de digression.

Dans le registre oral, celui de la conversation, Domitille Caillat, propose un système de «balisage multimodal ». À partir d'un corpus de conversations orales spontanées entre une mère et sa fille, l'auteur procède au repérage des indices fournis par le locuteur pour signaler des propos représentés au style direct. Elle analyse la manière dont un phénomène de "double énonciation" (Ducrot 1984) permet de mettre en scène simultanément le discours représenté qui assure la gestion du récit, et le discours principal qui permet la gestion de l'écoute. Une « combinaison d'indices en faisceau » (p. 75) fondés moins sur les choix lexicaux, verbaux par exemple, que sur la posture (ou « mimo-posturo-gestualité ») et la prosodie permettent l'identification de la « rupture illocutoire » liée à l'introduction d'un discours rapporté. L'étude de ces indices permet de comprendre comment la représentation du discours direct parvient à installer l'interlocuteur dans une position de spectateur.

De l'oral à l'écrit, la contribution de Sylvie Plane, Fabienne Rondelli et Christine Venerin, traite de la capacité d'enfants à mémoriser un récit et à le transcrire, à travers l'observation de la manière dont ils restituent un récit transmis oralement. Il s'agit d'un conte traditionnel gabonais raconté dans deux versions différentes, l'une utilisant le discours direct rapporté, l'autre le discours indirect. Les auteurs analysent comment lors des deux transcriptions, immédiates puis après une semaine, les enfants se focalisent alternativement sur la restitution des éléments narratifs (« dictum»), d'une part, et sur les modalités d'énonciation («modus»), d'autre part. Cette méthode permet d'identifier les diverses stratégies utilisées, en fonction de l'âge des enfants et du stade de la retranscription, pour marquer et restituer les diverses strates énonciatives qui structurent le récit.

16 L'ensemble des contributions ne manque pas de souligner les difficultés rencontrées pour un marquage clair des différents types de discours rapporté et surtout pour leur délimitation, leur clôture en particulier. Elles montrent également que ces obstacles font partie inhérente du genre abordé. Ainsi, les études consacrées à la fiction, notamment chez Godard et Mahfouz, permettent d'entrevoir l'intérêt pour le récit littéraire de maintenir un espace flou dans l'assignation de la parole. De même, l'analyse des comptes-rendus de réunions prouve l'importance, à côté des indices linguistiques, de la connaissance des éléments contextuels liés au genre pour l'interprétation du discours rapporté. Toutes, en fonction du type de discours traité, montrent combien le repérage des différentes «strates énonciatives » est essentiel pour l'appréhension d'une logique textuelle et l'élaboration de stratégies de lecture efficaces.

17 Le projet qui sous-tend l'ouvrage, de la recherche linguistique à son application pour l'élaboration collective d'un logiciel didactique, autour d'un objet de recherche très spécifique, lui confère ses qualités de cohérence en même temps que d'ouverture à des genres aussi divers que parfois inattendus. Ce qui fait tout l'intérêt de la lecture de cet 
ouvrage, dont la portée dépasse largement le monde des spécialistes du discours rapporté.

\section{BIBLIOGRAPHIE}

Authier-Revuz, Jacqueline. 2004. La représentation du discours autre : un champ multiplement hétérogène. In Lopez Munoz, J. M., S. Marnette \& L. Rosier. Le discours rapporté dans tous ses états. Paris : L'Harmattan.

Bakhtine, Mikhail. 1970. La poétique de Dostoïvski. Paris : Éditions du Seuil.

Carel, Marion et Oswald Ducrot. 2009. « Mise au point sur la polyphonie ». Langue française 4, 3343.

Dubois, Jean et Françoise Dubois-Charlier. 1993. Dictionnaire électronique des verbes français. Paris : Larousse. Disponible sur <http //www.modyco.fr>.

Ducrot, Oswald. 1984. Le dire et le dit. Paris : Les éditions de Minuit.

\section{NOTES}

1. Téléchargement gratuit <http://www.jeanlucminel.fr/navitexte.html>.

\section{AUTEURS}

\section{GENEVIĖVE BORDET}

Université Paris Diderot, CLILLAC-ARP.gbordet@eila.univ-paris-diderot.fr 